\title{
All the right moves
}

\author{
Dispelling stigmas and highlighting benefits of the Nature Research manuscript transfer service.
}

$\Lambda$ uthors whose papers have been declined by Nature Methods may have noticed a paragraph in the e-mail from their editor inviting them to explore the "manuscript transfer portal." By following the link, authors can seamlessly transfer their paper and associated submission information to another journal in the Nature Research family with just a few clicks. If the paper has gone through peer review, the reviews, reviewers' identities and confidential comments, and the editor's decision letter will also be transferred.

Anecdotally, many authors have told us that they think utilizing the transfer service hurts their chances of being published at another journal-that the editors at the receiving journal will be negatively influenced by their knowledge that the paper was already rejected elsewhere. But in reality, it depends on how similar or different the scopes and editorial standards of the journals are. In many cases, a paper that is not a good fit for one journal might be an excellent fit for another in the Nature family (which, besides Nature, includes the other Naturebranded titles, Nature Communications, Communications Biology, Communications Chemistry, and Communications Physics, as well as Scientific Reports and several academic partner journals).

When a paper is rejected by Nature Methods, whether before or after review, the option to transfer to another journal will generally be conveyed by the editor in one of three ways.

Transfer link with a guarantee: For strong papers that are just a bit borderline (or out of scope) for Nature Methods, we may consult with editors at one or more Nature-family journals about their interest in receiving a transfer. We most often consult with Nature Communications and Communications Biology. We share the paper and some notes about it. If it has been peer reviewed, we share the reviews, reviewers' identities, and any confidential remarks from the reviewers. Authors can opt out of such consultations by clicking on a box at the submission stage (more on this below). When authors receive a rejection letter with a guarantee that another Nature-family journal will send the paper for peer review, consider a revision, or even accept the paper without further rounds of review, they can trust that the editor of the receiving journal will make good on his or her word. If we know that a particular editor will be assigned to handle the paper at the receiving journal, we include his or her name as a contact point.

Transfer link with a recommendation: In this case, Nature Methods may recommend a more suitable journal to transfer the paper to, but we make no guarantees that the recommended journal will consider the paper. All Nature Research titles are editorially independent, which means that the individual editorial teams make their own decisions about what to publish. However, we do have a relatively good sense of the scopes and editorial standards at our sibling titles; in recommending transfer, we aim to give sound advice to authors about the surest route to publication in a Nature-family journal, not set them up for a second rejection.

Transfer link provided but no recommendation: If a link is provided but no recommendation to a particular journal is given by the Nature Methods editor, this generally indicates that we think the paper is scientifically sound, but we are not confident in recommending an alternative journal that would be a good fit.

As always, the decision of where to submit your work lies with you; authors are not bound by an editor's transfer recommendation. Authors may transfer to a journal other than the one recommended. Authors may choose not to transfer but rather to submit afresh to a different journal. But if your paper has been peer reviewed, a transfer might allow you to publish faster than if starting anew, and can help you avoid new rounds of review with new reviewers who might raise different concerns. This also avoids duplication of effort on the part of reviewers. That said, it is sometimes necessary for the receiving journal to assign a new reviewer, for example, to evaluate an aspect specifically relevant to that journal's particular scope.

Before transferring, authors should consider the journal's audience: for example, do you want to emphasize a new method, or the results? Do you want to publish in a journal with a very broad readership, such as Nature Communications, or do you want to reach a specific target audience? If your paper is written to appeal to a journal with a particular scope, you may want to consider whether some judicious rewriting of the cover letter-and perhaps of the manuscript itself-could improve your chances at a different journal.

As mentioned above, authors may opt out of the editorial consultation process, which means that we will not share any details of your paper with other Naturefamily journals. Authors should be aware that opting in does give us leave to discuss your paper with other editorial teams. However, we rarely consult with other journals about their interest in a paper unless we feel it is strong and scientifically sound, and therefore opting in more often than not works in authors' favor-we want to help good papers find a suitable home in the Nature family. Moreover, the editor at that receiving journal may provide some feedback, for example, about which comments raised by the reviewers are necessary to address and which are notagain, saving authors time, as well as effort.

Unless you have opted out of editorial consultations with other journals, a lack of a recommendation to a different journal post-peer review usually means that the reviewers' technical concerns are sufficiently serious that we do not think that another journal will be strongly interested in considering the paper. You are still welcome to try transferring the paper to another journal, along with a rebuttal letter that explains how you plan to address the reviewers' concerns. But in our experience, when serious technical issues have been raised, authors are usually better off first addressing the most egregious concerns and then starting the manuscript submission process afresh at a different journal. If in doubt, you can send an e-mail to ask your Nature Methods editor for advice.

For more information and FAQs about the manuscript transfer service, see our guide for authors online. We hope the transfer service is a useful tool for our authors, and we welcome your feedback.

Published online: 30 April 2019 https://doi.org/10.1038/s41592-019-0424-9 